\title{
Le glissement du Friolin, en Savoie : mesures par imagerie numérique
}

F. GIRAULT

BRGM, BP 6009,

Orléans Cedex

\section{B. GOGUEL.}

Coyne et Bellier,

9 allée des Barbanniers, 92632 Gennevilliers Cedex

\section{J.-P. ASTE}

JPA Consultants, 505, avenue du 8 mai 1945 , 69300 Caluire
Les techniques de traitement d'images utilisées en télédétection rendent possible une approche nouvelle de l'étude des versants instables.

Leur application au versant Est du Friolin, siège d'un mouvement majeur en 1982, a permis de confirmer objectivement l'interprétation des désordres observés : glissement de type rotationnel, par lequel une dizaine de millions de $\mathrm{m}^{3}$ de rocher sont descendus quasiment en bloc, d'une cinquantaine de mètres d'altitude. L'analyse photogrammétrique de deux couples de photographies aériennes scannérisées caractérise le glissement et les changements survenus dans la forme du versant. Le repérage de points images (pixels) homologues sur les scènes d'avant et après glissement révèle une déformation significative du volume glissé : écrasement dans le sens de la pente, insoupçonnable autrement.

\section{The mount Friolin slide in Savoy : Digitized imagery measurement}

The image processing techniques used in remote sensing provide a new approach for studying unstable slopes. Their application to the eastern slope of Mt Friolin, where major movement occured in 1982, has provided objective confirmation of the interpretation of the disorders observed, i.e, rotational slide in which ten millions cubic meters of rock in practically a single piece have slid some fifty meters vertically.

Photogrammetrical analysis of the two pairs of digitized aerial photographs characterizes the slide and the changes which have taken place in the shape of the slope. Identification of similar pixels on $\approx$ before $x$ and $"$ after $n$ pictures reveals a significant deformation in the volume that has slid, i.e. with " concertina w condensation in the direction of the slope, something which would not otherwise have been imagined.
} 


\section{Caractéristiques du site et historique}

L'instabilité qui affecte la face est de la Pointe de Friolin, au-dessus de la vallée de Peisey-Nancroix, dans le massif de Bellecôte, en Vanoise, est un phénomène naturel majeur dont une analyse a déjà été publiée dans ces colonnes (B. Goguel, 1989). Pour la commodité de l'exposé, nous reprendrons ici la même toponymie pour désigner les différentes parties de cette face.

Rappelons brièvement que le Friolin, constitué de gneiss et de schistes, comporte un plateau sommital extrêmement fissuré, vers $2700 \mathrm{~m}$ d'altitude. I] présente sur son versant oriental, sur une largeur de près de $600 \mathrm{~m}$, une succession d'éperons rocheux et de couloirs plus ou moins bien individualisés avec une pente moyenne avoisinant $45^{\circ}$ sur plus de $300 \mathrm{~m}$ de dénivelée (Fig. 1). Cet ensemble repose en discordance, vers $2400 \mathrm{~m}$, sur des gypses et cargneules du Trias dont les pentes s'adoucissent progressivement jusqu'au Grand Plan (alt. 2150 ).

La dissolution progressive du soubassement de gypse déstabilise les escarpements supérieurs du Friolin. Un risque d'éboulement majeur fut signalé là dans les années soixante par le Professeur Jean Goguel. Il installa divers repères de surveillance sur le plateau sommital et institua une surveillance photographique, spécialement renforcée à partir de la " crise » de 1982, où d'abondantes chutes de blocs se produisirent dans le versant.

La comparaison de nombreuses photos prises au fil des années par Jean et Bernard Goguel a permis d'établir que la crise de 1982 correspondait à un gigantesque glissement, par lequel tout l'escarpement rocheux est descendu en bloc sur plus de $50 \mathrm{~m}$ de dénivelée. Fait remarquable, la masse rocheuse ainsi déplacée paraissait intacte, exempte de déformations significatives sur plus d'une quinzaine d'hectares, hormis quelques desquamations locales.

L'analyse des photos révélait aussi d'importantes déformations dans les terrains du Trias, à la base de l'escarpement rocheux. Etaient ainsi mis en évidence, plus particulièrement (voir Figure 3), un bourrelet au débouché du «Couloir Sud ) ( ( le bourrelet actif ») et un arrachement dans la partie centrale du pied de la face ( l'arrachement inférieur $»$ ).

La surveillance visuelle et photographique n'a décelé aucume reprise des événements, après ceux des années 1982 à 1985 révélés en 1988, Il importait cependant de tirer tout le parti possible des couvertures aériennes disponibles à I'TGN, pour appréhender la cinématique des mouvements en vue d'éclairer l'appréciation des risques rémanents.

\section{Nouveaux outils d'analyse et principe de la démarche}

Les photographies aériennes de type IGN sont habituellement exploitées pour le suivi et l'analyse de l'évolution de sites affectés par des mouvements de terrain, en raison de la "mémoire » que représentent ces clichés, des examens comparatifs qu'ils autorisent, et de la perception du relief - donc de la morphologiequ'ils permettent en vision stéréoscopique. L'information perçue est cependant essentiellement qualitative, l'aspect quantitatif ne pouvant être abordé que par une analyse photogrammétrique mettant en jeu des moyens beaucoup plus élaborés et donc plus onéreux.

Or, depuis le début des années 90 , les techniques de traitement d'image se sont largement développées, notamment dans le domaine de la photogrammétrie, de sorte que l'on peut obtenir maintenant des modèles numériques de terrain (MNT) et des orthoimages à maille très fine, de l'ordre du mètre.

Une orthoimage est une image redressée, corrigée de toutes les déformations dues à la prise de vue et au relief, qu'elle présente en plan comme une carte. Elle est établie à partir d'un couple de photographies en noir et blanc ou en couleurs, dont elle restitue toutes les nuances. Chaque point image est caractérisé par ses coordonnées et sa couleur.

La société ISTAR - Imagerie Stéréo Appliquée au Relief-propose ces produits géoréférencés, dérivés de l'analyse automatique par autocorrélation de couples stéréographiques numérisés de photographies aériennes. Initialement, la méthode avait été développée pour l'exploitation d'images SPOT; elle a été, par la suite, étendue à la photographie aérienne.

L'analyse présentée ici est basée sur la comparaison des états de surface et des morphologies du versant est du Friolin, décrits par des fichiers numériques issus du traitement des clichés IGN de 1970 et de 1986 (échelle $1 / 30$ 000). La qualité des clichés, et le fait que cette étude ait constitué la première exploitation de ce type de données, expliquent que les MNT et les orthoimages aient été restitués aux pas respectifs de $4 \mathrm{~m} \times 4 \mathrm{~m}$ et de $2 \mathrm{~m} \times 2 \mathrm{~m}$, avec une incertitude de $3 \mathrm{~m}$ sur le calage altimétrique absolu.

Les autres cas traités depuis montrent que l'on peut obtenir, selon la qualité et l'échelle des clichés utilisés, et selon qu'il y a eu ou non une stéréopréparation, des MNT plus fins ( $2 \mathrm{~m} \times 2 \mathrm{~m}$ avec une incertitude de $3 \mathrm{~m}$ sur le calage altimétrique, pour des clichés à l'échelle du 1/8000 par exemple). 


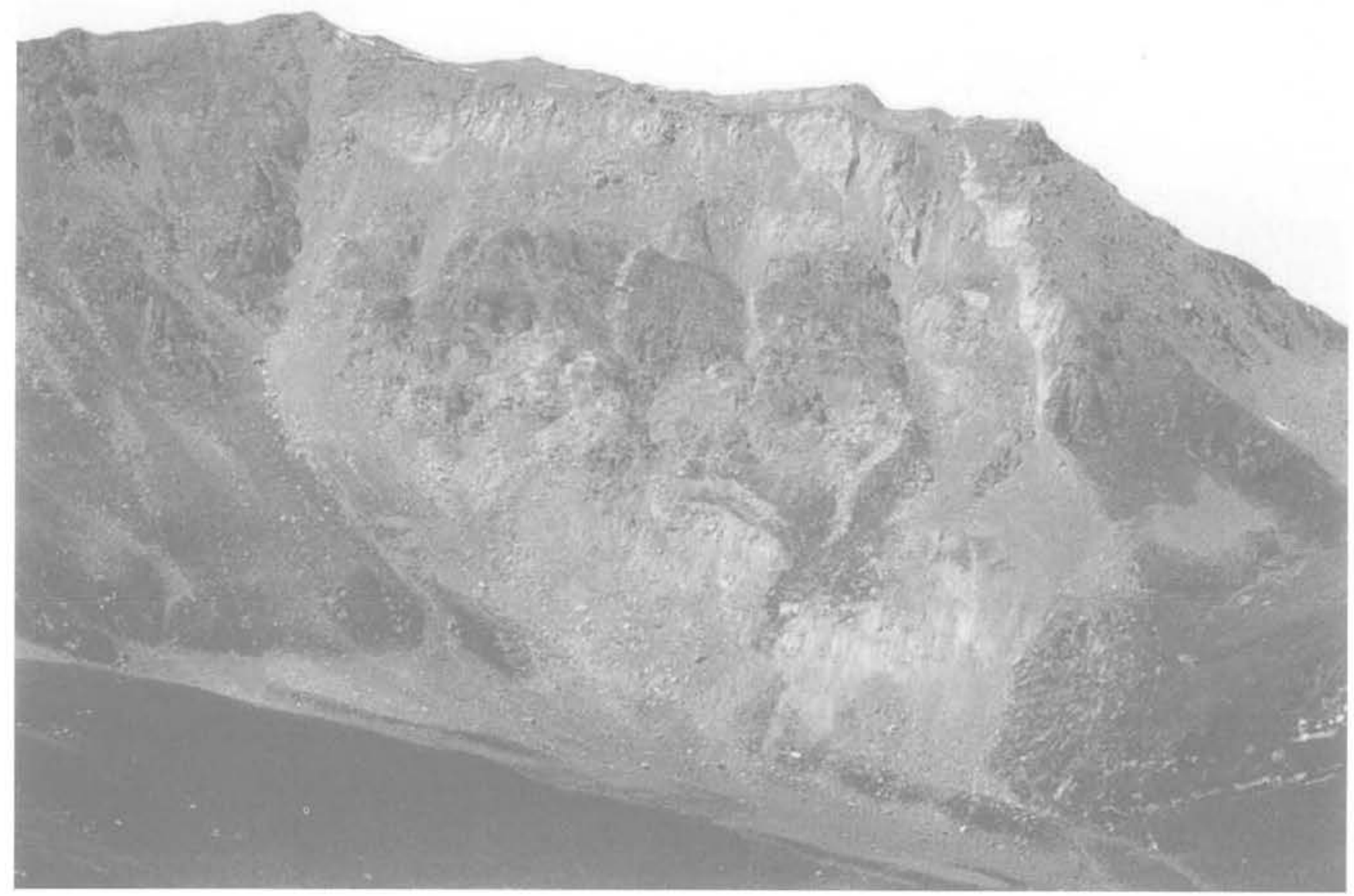

FG.1 Le versant est du Friolin : vue prise en 1989 de $5 \mathrm{~km}$ au NE (depuis alt. $2500 \mathrm{~m} \mathrm{env}$.).

The east face of Mount Friolin, taken in 1989 from $5 \mathrm{~km}$ distance North-East (at elev. $2500 \mathrm{~m}$ approx.).

\section{3}

\section{L'exploitation numérique des clichés aériens : étude de l'évolution du versant}

L'image des différences entre deux MNT montre à la fois qualitativement et quantitativement l'évolution morphologique du versant considéré. Cette image, dans laquelle chaque pixel a une valeur correspondant à la variation altimétrique - positive ou négative - de la surface de terrain qu'il représente, constitue une carte de la composante verticale du champ des déformations superficielles du site (Aste et Girault, 1992; Girault, 1992). Elle révèle ainsi, dans sa continuité spatiale, l'extension de la zone de désordres, les zones déprimées ou soulevées qui sont l'expression de surface de phénomènes profonds que les moyens traditionnels ne permettent pas de mettre en évidence.

L'imagerie électronique en couleurs aide à percevoir les changements intervenus sur le site considéré. Au Friolin, cette image indique que, sur toute la largeur du versant, la partie haute est déprimée, alors que la partie basse a gonflé. Elle révèle, sous "l'arrachement supérieur », un abaissement de $50 \mathrm{~m}$ de toute la bordure du plateau sommital, ainsi que l'évolution du ( Grand Couloir NE » et du « bourrelet actif » au débouché du "Couloir Sud ». Il apparaît aussi que « l'arra- chement inférieur » ne correspond pas à un effondrement, comme le suggérait une première hypothèse. mais à un bornbement.

\section{Volume des déformations superficielles}

L'extraction de données quantitatives permet d'estimer avec une bonne précision la surface et l'amplitude verticale, donc le volume des déformations superficielles. Les calculs donnent, pour la partie strictement rocheuse du versant, une dépression de 2,5 millions de $\mathrm{m}^{3}\left(\mathrm{hm}^{3}\right)$, et un gonflement à la base de l'escarpement de $0,9 \mathrm{hm}^{3}$. Plus bas, le gonflement des terrains du Trias atteint presque $2,3 \mathrm{hm}^{3}$. Ces chiffres seront discutés un peu plus loin.

Mais, si ces données suffisent à prouver l'importance du phénomène, c'est surtout l'étude du comportement de points remarquables de cette face qui apporte des enseignements sur la cinématique et les mécanismes du mouvement. 


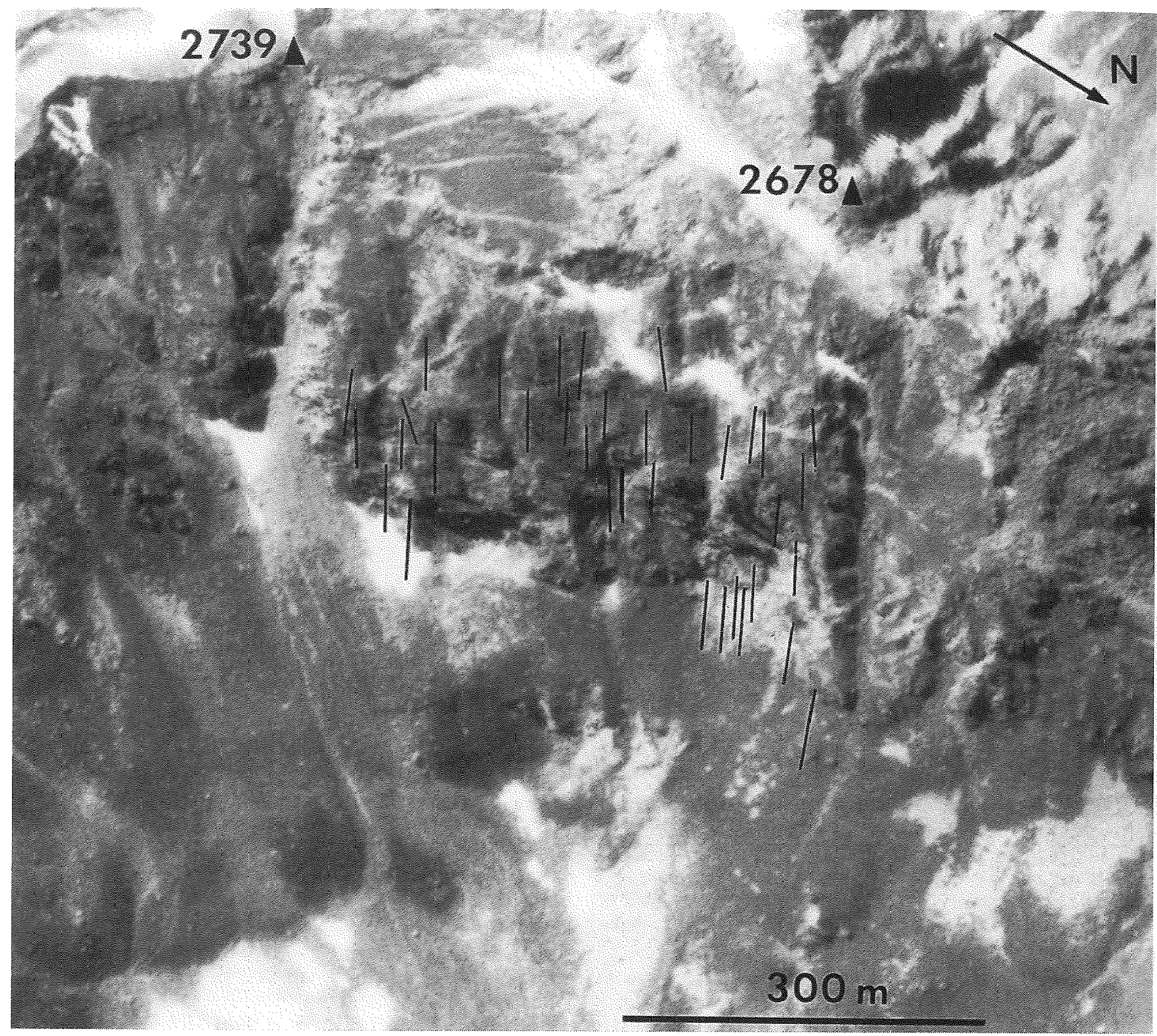

HG.2. Orthoimage de 1970 et déplacements de 1970 à 1986 (composantes horizontales seules représentées). 1970 orthoimage and displacements from 1970 to 1986 (horizontal components only).

5

\section{Recherche et analyse de vecteurs de déplacement}

Le versant ayant eu un comportement quasi monolithique et gardé son apparence physique, de nombreux traits physiographiques se retrouvent sur les deux orthoimages de 1970 et de 1986 (Fig. 2 et 3). remarquables ont pu être identifiés formellement sur ces orthoimages et constituent autant de ( pseudorepères ) de surveillance topographique. Ils ont été utilisés pour déterminer, à partir de leurs coordonnées géographiques avant et après glissement, des vecteurs de déplacement. L'évolution du versant peut ainsi être décrite objectivement.

Ces vecteurs présentent des caractéristiques particulières. En plan (Fig. 2) leur parallélisme est presque parfait, l'orientation moyenne est $\mathrm{N} 60^{\circ} \mathrm{E}$. 


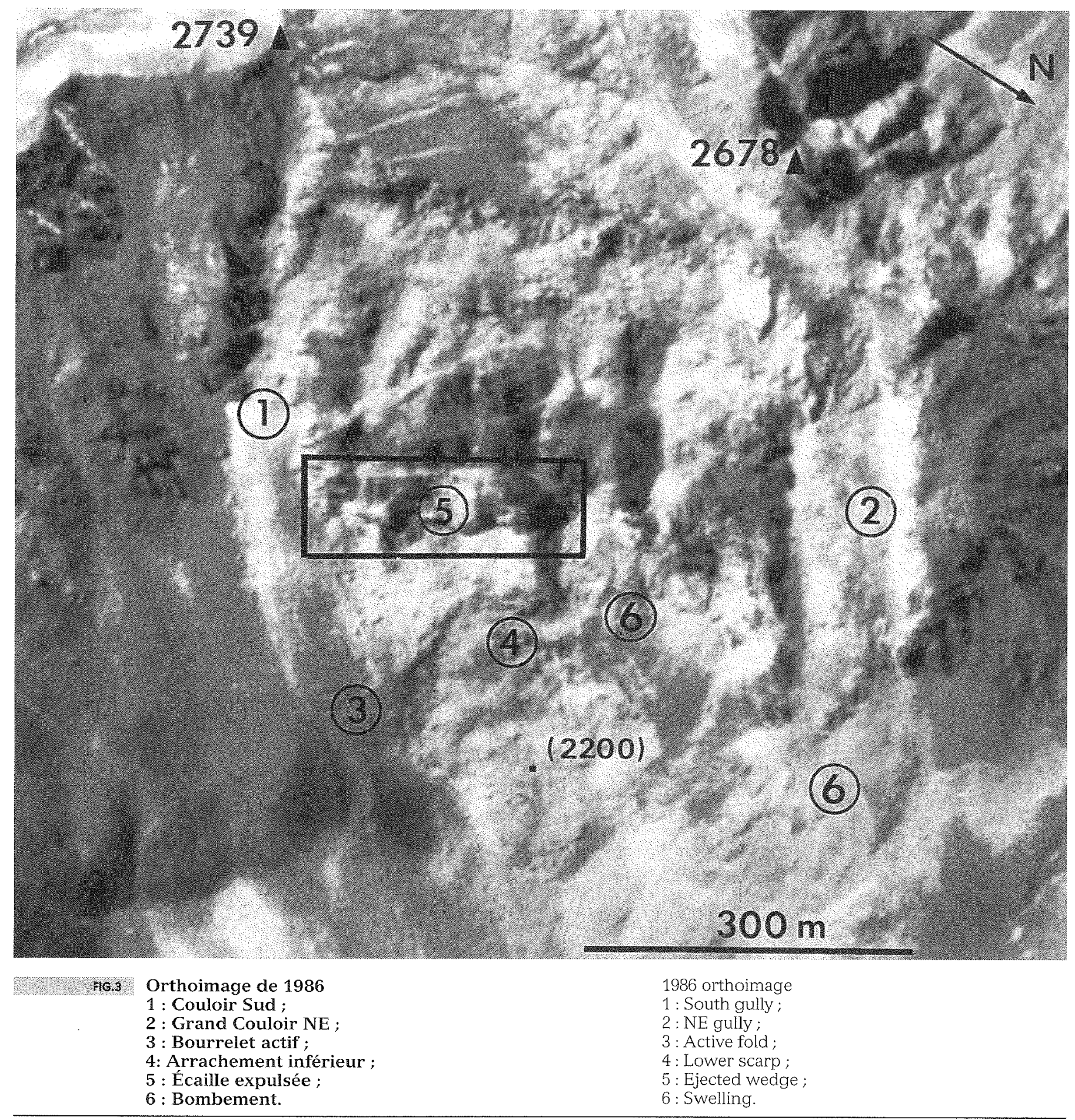

Leurs inclinaisons (Fig. 4-a) varient directement avec l'altitude du point de départ, allant de $50^{\circ}$ environ sous l'horizontale (plus raide que le versant) en haut de l'escarpement rocheux, à 20 ou $30^{\circ}$ en bas (sortant du versant).

Leurs composantes verticales (Fig. 4-b) varient dans le même sens, d'une soixantaine de mètres vers la cote 2600 à une vingtaine de mètres vers la cote 2400 . Toutes ces indications correspondent à un mouvement d'ensemble de type glissement rotationnel.
Les modules, ou longueurs des déplacements effectifs (Fig. 4-C), apparaissent eux aussi étroitement corrélés avec l'altitude : ils passent de $80 \mathrm{~m}$ vers 2600 , à seulement (!) $60 \mathrm{~m}$ vers 2 400. Même si l'escarpement rocheux paraît être descendu en bloc monolithique, il s'est en fait raccourci d'une vingtaine de mètres dans le sens de la pente, subissant un écrasement de 5 à $10 \%$ dans le sens du mouvement. Cette déformation intrinsèque du volume glissé complique l'estimation de la forme de la surface de rupture, alors qu'on ne connaît que les déplacements superficiels. 


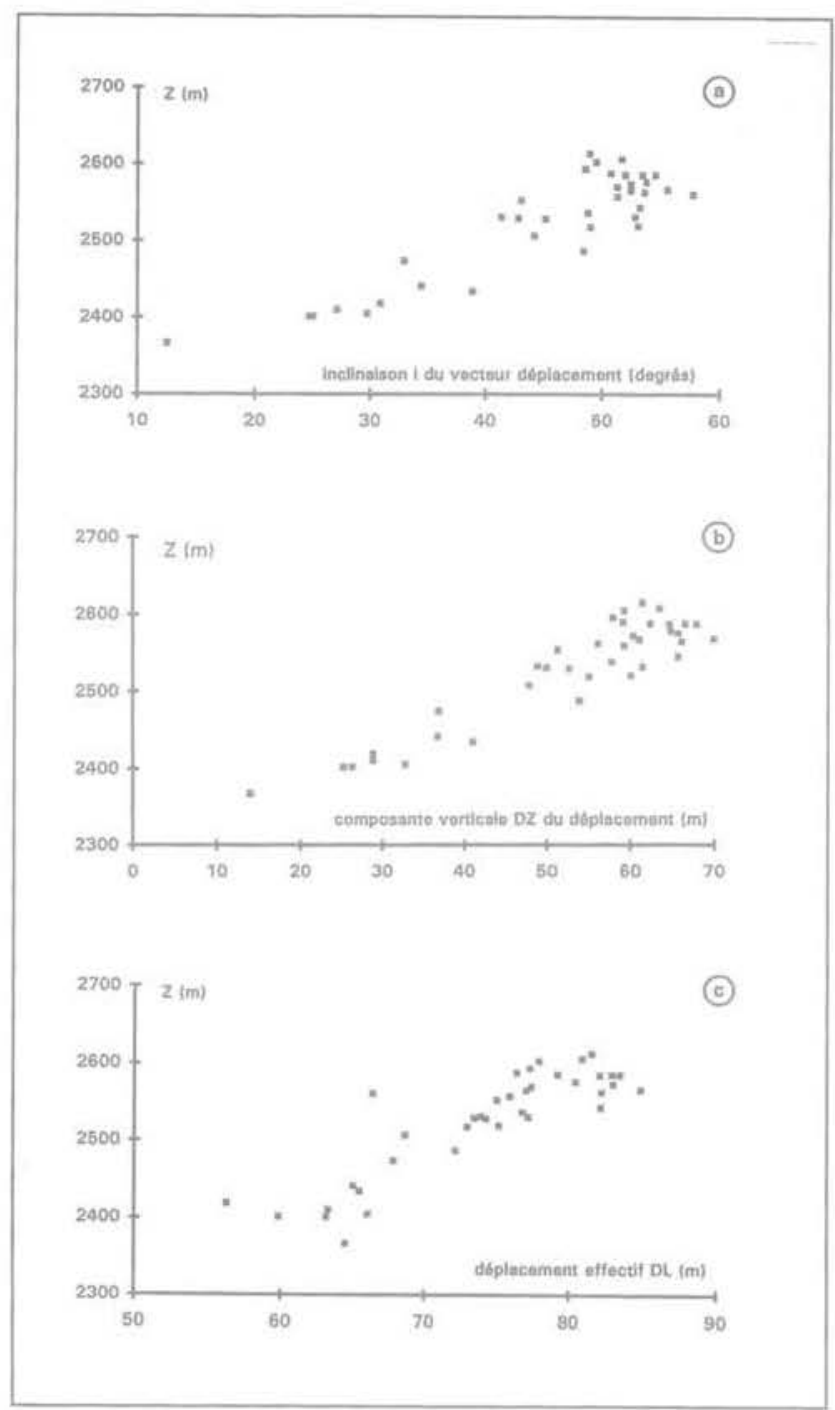

FIG.4 Relations déplacement/altitude, pour 37 points :

Relations displacement versus elevation for 37 points :

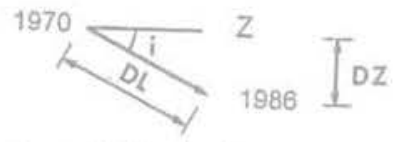

a - inclinaison (inclination) i

b - composante verticale (vertical component) DZ

c- longueur (length) DI

Il serait tentant d'envisager un cylindre de rupture de rayon variable, passant progressivement de l'ordre de $800 \mathrm{~m}$ en haut du versant à une valeur moitié moindre dans le bas : ce serait le « glissement spirale $»$. Mais le glissement circulaire compressif rend aussi bien compte des observations.

Il aurait été souhaitable de trouver des points supplémentaires, en particulier dans la partie sud du versant, à la base de l'escarpement et dans les terrains du Trias du pied. Mais la qualité des images ne l'a pas permis. Des travaux photogrammétriques complémentaires devraient pouvoir contribuer à surmonter cette difficulté.

\section{Étude de profils topographiques comparatifs}

L'un des grands intérêts des MNT est qu'ils se prêtent facilement à l'extraction de nombreux profils topographiques. Sur le Friolin, quatorze profils comparatifs ont été examinés. Il est possible d'identifier, sur la plupart d'entre eux, l'arrachement de la zone sommitale et les bourrelets au pied du versant. On peut esquisser une surface cylindrique de rupture ; elle sortirait au pied entre $2200 \mathrm{~m}$ et $2300 \mathrm{~m}$ d'altitude. C'est là que le versant présente des particularités morphologiques comme le « bourrelet actif $»$ et « l'arrachement inférieur ». Le volume mis en jeu dans le glissement, large de $450 \mathrm{~m}$, serait alors compris entre 10 et $15 \mathrm{hm}^{3}$.

Des analyses photogrammétriques complémentaires, affinées, mettent en évidence un trait particulier du gonflement du versant, à la base de l'escarpement. rocheux. On observe là, dans la partie méridionale, vers la cote 2450 , l'expulsion d'une écaille, ou coin rocheux, d'une cinquantaine de mètres de hauteur et de $200 \mathrm{~m}$ de largeur. La figure 5 schématise l'interprétation suggérée, appuyée par ailleurs sur des observations directes de terrain. On peut ainsi considérer que sur une emprise de près de 38 ha, les creux et les bourrelets ont des volumes à peu près équivalents (respectivement $2,5 \mathrm{hm}^{3}$ contre 2,3), l'écaille et le basculement de l'escarpement rocheux contribuant à environ $0,9 \mathrm{hm}^{3}$ de gonflement supplémentaire.

Globalement, le bilan des mouvements du sol fait apparaître un gonflement de $3,2-2,5=0,7 \mathrm{hm}^{3}$, c'est-àdire de l'ordre de $6 \%$ du volume glissé. Vu de près, le massif rocheux présente une structure cen piles d'assiettes » tellement aérée par de nombreuses fractures que la notion de dilatance s'y conçoit aisément..

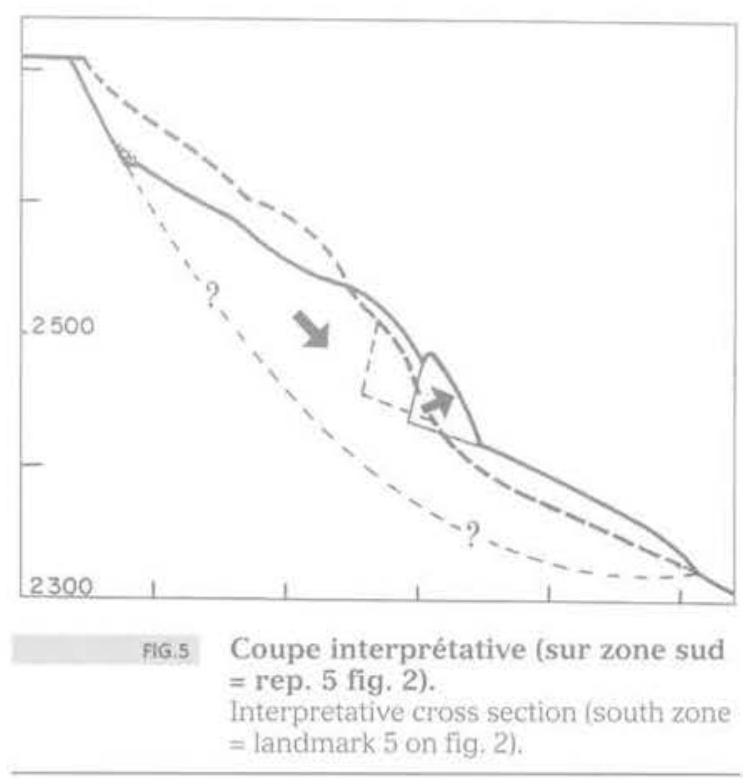




\section{Conclusions}

L'exploitation par voie numérique de photographies aériennes a permis de prouver la justesse et la validité des observations précédemment publiées, à savoir que le versant est du Friolin s'est affaissé, en 1982-1985, de plus de cinquante mètres.

La méthode utilisée fournit :

- une carte de la zone accidentée mettant en évidence l'évolution morphologique du versant,

- les caractéristiques de ce glissement de très grande ampleur, dont le volume est estimé entre 10 et 15 millions de $\mathrm{m}^{3}$; avec, en particulier, la description du déplacement de points remarquables, c'est un atout essentiel pour la mise en évidence et la compréhension des mécanismes de l'évolution du versant. Contrairement aux apparences, celui du Friolin n'est pas vraiment monolithique : au cours d'un mouvement de glissement rotationnel de grande ampleur, vers le NNE, il a subi un raccourcissement de 5 à $10 \%$ dans le sens de la pente.

Cet exemple, étudié grâce à des documents d'archives, illustre l'apport essentiel de la photographie - même au format $24 \times 36$ - et de l'imagerie numérique pour l'analyse et la caractérisation de l'évolution de mouvements de terrain de grande ampleur. Sans atteindre un degré de précision très élevé puisqu'elle est limitée par la taille du pixel et l'incertitude sur le calage en z des MNT, la méthode proposée s'inscrit en complément des méthodes traditionnelles d'auscultation des versants instables.
L'exploitation de clichés stéréophotogrammétriques à grande échelle, pris du sol ou d'hélicoptère, devrait permettre d'améliorer et de généraliser ce type d'approche à l'auscultation de mouvements de moins grande ampleur.

Au Friolin, il n'y a aujourd'hui encore aucun dispositif de surveillance institué. L'importance de l'événement de 1982-1985 et l'état de fracturation du plateau sommital et du versant sont pourtant très impressionnants. Ils devraient inciter à la plus grande prudence : sans parler d'une reprise du glissement qui pourrait ètre catastrophique, des chutes de blocs sont à envisager qui pourraient aller au-delà des limites des éboulements actuels. Une surveillance de ce grand versant accidenté, quasiment impraticable, pourrait allier un suivi topométrique - avec mise en œuvre au cours de visites périodiques du système GPS de positionnement par satellites - et des analyses photogrammétriques fines.

\section{8}

\section{Remerciements}

Cet article est la publication scientifique n 92021 du BRGM ; il a été réalisé dans le cadre d'un projet financé sur crédits de recherche du BRGM, avec la participation gracieuse de Coyne et Bellier.

This is the BRGM contribution $n^{\circ} 92021$; this work was financially supported by a BRGM research project, with Coyne \& Bellier collaboration.

\section{$\overline{\text { Bibliographie }}$}

Aste J.P., Girault F. - GIS, SPOT, DEM and morphology of major land movements. 6 th Int. Conf. on Landslides, Christchurch, New Zeland, 1992.

Girault F, - Auscultation de versants instables par imagerie numérique ; thèse d'ingènieur CNAM, Paris, 193 p, 1992.

Girault F. - The use of numerical imagery and photogrammetric methods for the monitoring of unstable slopes, Proc. of a conf. held on $8 / 9$ march 1994 at the
Royal Society, London, UK entitled « Natural Hazards and Remote Sensing $x$, ed. by G. Wadge, pp. 51-53.

Girault F., Fleury B, - Photogrammetry and digital imagery : application to measuring and defining deformations in unstable slopes, Proc. $1^{\text {tt }}$ Int. Airborne Remote Sensing Conf. and Exhibition (Strasbourg, France), 1994, vol. IIl, pp.198-204. Goguel B. (1989) - Le glissement du Friolin, en Savole : un mouvement majeur révélé par les photographies. Revue Française de Géotechnique $n^{2} 48,1989$, p.55-64.

Goguel B. - Le glissement du Friolin : un mouvement exceptionnel sans dislocation de la masse rocheuse. $7^{\text {th }}$ ICRM, Aachen 1991, thème 3.

Habib P. - Discussion : mouvements de terrains en Tarentaise, Revue Française de Géotechnique, $n^{\circ} 53$, 1990, p. 71-74. 